\title{
DIDÁCTICA DE LA FILOSOFÍA
}

Julio Perelló, sdb

Universidad Politécnica Salesiana - Quito

Apuntes del Profesor

PUCE - Quito - 1992

Toda enseñanza para que cumpla con sus objetivos debe servirse de la Didáctica $(=$ metodología $=$ camino) correspondiente.

Parece que esta afirmación cobra mayor importancia en la enseñanza de la Filosofía. Las transformaciones operadas últimamente en nuestro mundo por los acontecimientos políticos, sociales y científico-técnicos han colocado como en situación de crisis permanente a la Filosofía y, por consiguiente, a su enseñanza:

- el desprestigio de todo saber que no pueda someterse al control de la experimentación y de la verificación;

- igualmente todo saber que no tuviere una aplicabilidad inmediata y eficiente;

- el descrédito de toda ciencia que se presenta con rasgos de dogmatismo y con desvinculación de las necesidades fundamentales del hombre para poder vivir con satisfacción;

- el desarrollo de los "mass media", como extraordinario condicionador de alienación personal, de 
consumismo y conformismo, de automatización y acriticidad;

- además, la escasez de diálogo entre distintas escuelas, el esoterismo, el gusto por el conceptualismo retórico, la oscuridad del lenguaje, etc., son otros tantos elementos que minan la seriedad de la Filosofía.

Si el objetivo del estudio de la Filosofía es el "enseñar a pensar", se comprende fácilmente que en nuestra sociedad pragmatista y superficial, se hace difícil el capacitar al alumno a la comprensión profunda de cualquier información, a la precisión rigurosa de su análisis, y a la formación crítica de una valoración ponderada.

De allí que, el estudiar un método de enseñanza de la Filosofía es fundamental para quien se sintiere llamado a ser profesor en esta disciplina.

El conocimiento de esta DIDÁCTICA DE LA FILOSOFÍA, después de una breve introducción, contemplará dos partes básicas:

- Primera parte: Didáctica general

Aquí se expondrán los principios generales de toda Didáctica de la enseñanza-aprendizaje, principalmente los factores que condicionan su eficiencia, y las distintas modalidades de la enseñanza. Esta parte concluirá con la exposición de las distintas clases de lección en el aula.

\section{Segunda parte: Didáctica de la Filosofía}

Aquí se expondrán las metodologías propias de la enseñanza de esta disciplina metafísica. 


\section{INTRODUCCIÓN}

La Didáctica de la enseñanza es un condicionador extrínseco de la formación intelectual. Dentro del cuadro epistemológico de las Ciencias de la Educación, pertenece a la Pedagogía especial o metodología.

- la Pedagogía General estudia en forma sistemática los principios generales que se refieren al hecho educativo: Fin de la educación, Educar-educando, Valores educativos, Encuentro educativo, etc.

- la Pedagogía Especial estudia, también en forma sistemática, las distintas formaciones (sectoriales) del hombre, que se unifican en el fin de la educación: formación física, intelectual, moral, religiosa, social, etc.

El estudio de la Didáctica forma parte de la "formación intelectual".

La Didáctica, a su vez, puede ser:

- General: estudia la Teoría de la Teoría; es la ciencia llamada a dar los principios fundamentales de la enseñanza para que ésta pueda cumplir su finalidad de ayudar al aprendizaje del alumno.

- Especial: estudia la Teoría de la Práctica; es la ciencia llamada a teorizar la práctica docente desde distintos puntos de vista:

- del proceso: kinder, primaria, secundaria, universidad.

- de las asignaturas: ciencias exactas, positivas, filosóficas.

- de la normalidad del sujeto: normal, deficiente, retardado. 
- Didaxis: es al Práctica de la Teoría; es la docencia "en acto". Resulta de la síntesis del "estudio" y de la "experiencia" personal. En este sentido, ya no es ciencia, sino Arte.

De acuerdo a lo dicho hasta aquí, la Didáctica de la Filosofía pertenece a la Didáctica Especial. Es una ciencia "normativa que saca sus fundamentos teóricos de la Filosofía y de las ciencias psicopedagógicas.

En nuestra reflexión haremos un estudio somero y suficiente sobre algunos principios básicos de la Didáctica General, para luego detenernos en la Didáctica especializada de las ciencias Filosóficas.

\section{PRIMERA PARTE: PRINCIPIOS BÁSICOS DE LA DIDÁCTICA GENERAL}

\section{Terminologia (=explicatio terminorum)}

Nos preguntamos: La Didáctica Catequística ¿cumple su cometido como "enseñanza", o como "instrucción", o como "formación"? ¿ No lleva como finalidad al contribuir a la "educación" total de la persona del alumno?

Ahora queremos clarificar el significado propio de cada uno de estos términos.

- Enseñar (= in signo) significa presentar algo en "signos". En la naturaleza del acto de enseñar, el aprendizaje por parte del alumno no es exigitivo.

- Instruir (= in struere) significa "construir dentro". En la naturaleza del acto de instruir, el aprendizaje del alumno es exigitivo. 
- Formar: no basta con "informar" al alumno. La formación exige "darle forma". La inteligencia estará formada cuando disponga de hábitos mentales, de capacidades arraigadas para adquirir nuevos conocimientos, para criticar ideas y hechos, para elaborar personalmente datos y conceptos.

En síntesis: poseer la capacidad de pensar rectamente.

- Educar (= educare) significa "extraer", sacar de "dentro". Mente educada no es solamente la que razona rectamente, sino la que contribuye a dar a la persona la capacidad de obrar el bien con libertad (que es el fin de la educación).

En nuestro caso, la Didáctica Catequística debería culminar en una verdadera "educación de la persona" mediante una recta formación intelectual, empleando adecuadas metodologías de enseñanza-aprendizaje.

\section{Factores de la enseñanza-aprendizaje}

En toda Didáctica existen factores llamados "condicionadores" de la enseñanza-aprendizaje. Consideremos los principales y más significativos que pueden aplicarse luego a la Didáctica de la Filosofía.

A) Factores sujetivo-psicológicos

Nos referimos a los aspectos psicológicos del aprendizaje, como son: la globalización, la motivación y la autoactividad.

\section{- Globalización}

En el aprendizaje, el primer conocimiento normalmente no es el analítico, sino el global, estructural. 
Este primer conocimiento consiste en una percepción intelectual confusa, genérica e indiferenciada. Posteriormente el intelecto es llevado a percibir y comprender las partes del todo, los detalles de lo percibido globalmente.

Por globalización entendemos la capacidad natural que posee el hombre para captar simultáneamente el mundo exterior como un "todo indistinto", como un conjunto confuso, del cual diferencia uno u otro elemento bajo el influjo de determinados estímulos (necesidades, tendencias, intereses, sentimientos).

Si todo conocimiento nuevo, antes de ser "claro y distinto" es confuso y global, es natural que el primer momento de la enseñanza deberá consistir en una orientación genérica y globalizante. Algunos metodólogos, inspirándose en este principio de la globalización, articulan así los momentos del ritmo didáctico:

- momento global (=sincrético): es esencialmente intuitivo

- momento operativo (=analítico): es esencialmente activo, de búsqueda, de investigación, de análisis de las partes. El protagonista es el alumno: él es el que investiga y analiza.

- momento recapitulativo (=síntesis): es esencialmente activo unificante y de sistematización. El protagonista es el profesor: él orienta y guía al alumno para realizar la síntesis de lo analizado.

- Motivación

La motivación es la acción por la que se presentan a la persona del alumno objetos (=valores) capaces de satisfacer profundamente y en forma permanente sus genuinas tendencias naturales. 


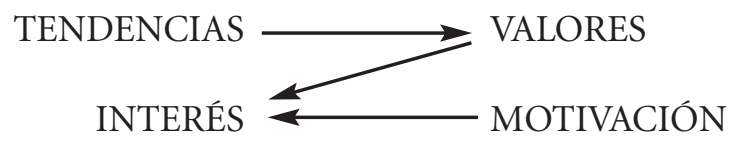

$\underline{\text { motivo }}$ (=motus) es lo que "mueve" a la determinación de un acto.

La motivación didáctica consiste en estimular mediante valores válidos el deseo de aprender o formarse intelectualmente.

La motivación didáctica implica fundamentalmente la percepción de la "relación" entre la tarea escolar y el objetivo por la que debe ser cumplida.

Desde el punto de vista metodológico es importante que los alumnos "conozcan" los objetivos básicos de la asignatura, del programa, del capítulo que debe estudiarse.

Lo que mueve al alumno a tener interés por determinados contenidos del aprendizaje es el "descubrir" que dicho contenido es un verdadero valor para la persona (que vale para él).

En realidad, son los valores los que mueven la voluntad y hacen tomar una decisión. La voluntad es libre, pero no indiferente.

\section{- Autoactividad}

Cuando el sujeto-alumno "descubre" la relación existente entre sus tendencias fundamentales y los valores que las satisfacen, entonces no necesita "ser obligado" a realizar o asimilar dichos valores; por su propia voluntad tiende a ellos. Nace de un impulso interior que está llamado a satisfacer exigencias de crecimiento perfectivo personal.

Esta actividad, que algunos llaman “espontánea”, es la autoactividad (= actividad movida desde "dentro"). 
En didáctica, es el mejor factor condicionante para un aprendizaje satisfactorio.

De esta "autoactividad" individual puede pasarse, metodológicamente a:

- una auto-actividad COLECTIVA: búsqueda e investigación grupal, elaboración y clasificación colectiva.

- un AUTOGOBIERNO democrático: escuela-ciudad, donde los alumnos (bajo la guía experta de los directivos) elaboran sus reglamentos, eligen sus jefes, dictan sanciones y vigilan la disciplina.

Las razones que justifican metodológicamente la autoactividad, pudieran ser:

- razón filosófica: si bien el profesor es la "causa ministerial" de la enseñanza-aprendizaje (= instrumental), con todo, la causa principal está en el mismo alumno; su capacidad intelectual es la "causa eficiente".

- razón psicológica: el alumno se experiencia colaborador, creador, actor; de lo contrario se sentiría condicionado, instrumentalizado y domesticado.

- razón social: la vida social del adulto exige la capacidad de iniciativa, de eficiencia personal, de creatividad. Tal capacidad se adquiere en la "experiencia concreta” de acciones autónomas y libres.

- razón de economía intelectual: la autoactividad capacita para poder continuar un aprendizaje por sí mismo (= autodidaxis) en el futuro. La metodología auto-activa proporciona más una capacidad, que un capital. 
A todo lo dicho debemos agregar que la autoactividad (fruto del interés-motivación) de ninguna manera suprime el esfuerzo personal del alumno, sino que le da sentido, consistencia y perseverancia.

B) Factores sujetivo-personales

Entre estos factores destacamos la personalidad del profesor y las capacidades del alumno.

- Personalidad del profesor

Los rasgos que vamos a presentar tienen su origen en los caracteres propios del filosofar como actividad humana. No existe auténtico profesor de filosofía que no sienta la exigencia del pensar filosófico. El profesor debe cultivar la reflexión crítica, el rigor de la investigación, el orden y la sistematización del pensamiento, la capacidad de penetración y de iluminación de la realidad. Estos rasgos son esenciales para el profesor de filosofía.

- actitud de búsqueda

Enseñar filosofía implica comprometerse con la verdad. Esto supone una actitud específica: apertura hacia los más variados horizontes del conocimiento, coraje ante los planteamientos de problemas fundamentales del hombre, conciencia crítica con capacidad de poner en tela de juicio cualquier certeza o convicción por segura que fuere. No siendo la verdad un pensamiento "congelado", el profesor no se reunirá a ser un mero transmisor de verdades acabadas, sino un permanente investigador, cuestionador y buscador de verdades objetivas.

Esto no significa que el profesor no deba tener convicciones personales o deba ocultarlas, sino que éstas deben ofrecerse como instancias abiertas. 
- actitud moral

A lo anterior deben unirse cualidades morales básicas: la honestidad intelectual, el respeto a la libertad de pensamiento y la tolerancia de las ideas. El profesor de filosofía no puede simular convicciones que no tiene, ni debe impedir la expresión de pensamiento de los alumnos, ni debe rechazar soluciones sólo por ser distintas a su enfoque personal. Su cometido no es el de adoctrinar, sino el de suscitar reflexiones críticas frente a doctrinas e ideologías. En este sentido, no debe ser ni militante ni propagandista. Se impone la reflexión crítica científica, coherente y sistematizada.

- lenguaje filosófico

El desarrollo del pensamiento crítico no puede prescindir del cultivo y dominio del lenguaje metafísico apropiado: concisión, propiedad, flexibilidad de la expresión; este lenguaje se opone radicalmente a la ambigüedad, vaguedad, oscuridad, rigidez y juego de palabras. Los filósofos usan términos y giros de lenguaje muy peculiares, que el profesor debe comprender con exactitud.

Muchos de los problemas que encara la filosofía tienen sus raíces en el mismo lenguaje filosófico, por no comprender su significado.

- cultura filosófica

Está demás decirlo, pero hay que insistir en al necesidad de una cultura filosófica segura y rica, actualizada y de primera mano. Enseñar filosofía no consiste en repetir nociones, sino en presentar a los alumnos un pensamiento elaborado por conocimientos, experiencias y reflexiones (a través de varios años) dentro de un proceso dialéctico que integra ideas y realidades en un constante juego de unificaciones y oposiciones. 
- Sicología del alumno

En la enseñanza-aprendizaje, el factor "alumno" es tan decisivo, como el factor profesor. Cuando el alumno carece de un conveniente nivel intelectual y de una relativa madurez personal, la enseñanza de la filosofía se hace imposible o, por lo menos, tropieza con grandes dificultades.

- escolaridad previa

Los vacíos de la formación básica en las distintas materias, particularmente las humanistas, comprometen seriamente la capacidad del alumno para el aprendizaje de la filosofía.

Los cursos de filosofía se colocan en los años terminales de la secundaria, porque suponen madurez intelectual y una gama de intereses apropiados.

Dentro de esta escolaridad el alumno debe haber adquirido una base lingüística suficiente. La carencia de esta base le dificulta la compresión de los textos de filosofía, y la elaboración y expresión de sus propios pensamientos.

\section{- madurez personal}

Una relativa madurez intelectual, afectiva y vital es fundamental para el alumno a fin de afrontar con claridad, amplitud y objetividad los temas filosóficos relacionados particularmente con al antropología, la ética y la metafísica.

Nos preguntamos iel adolescente en camino hacia la juventud está suficientemente maduro para los estudios filosóficos? 
- indudablemente tiene aspectos sicológicos positivos:

- capacidad de introspección y de reflexión frente al alumno que lo rodea;

- toma de conciencia de los problemas humanos que le circundan;

- abandono del egocentrismo y subjetivismo propios de la niñez;

- deseo de saber, comprender y dominar las circunstancias que pudieran suscitar en él, inseguridad personal.

- pero también existen aspectos negativos:

- tendencia al idealismo y a la ensoñación, unidos al despertar erótico y sensual, densos de emotividad y afectividad tan contrarios a la lógica matemática y al silogismo riguroso;

- cierto negativismo y escepticismo que le impiden ver la realidad con objetividad;

- el prurito de discutir y cuestionar, el afán de contradecir y negar, como consecuencia de una autoafirmación mal entendida.

- prejuicios sobre la filosofía

Muchos alumnos tienen una serie de prejuicios sobre la clase de filosofía y una cierta predisposición al rechazo, debido a las informaciones que reciben de sus compañeros. Por otra parte se cuestiona la utilidad de la asignatura para su vida futura y para su trabajo. El alumno sospecha que va a recibir unos conocimientos abstractos y difíciles de comprender.

Lógicamente, el profesor deberá tener en cuenta todos los aspectos subjetivos del alumno, para tomar conciencia de que la enseñanza de la filosofía no es fácil. Las modalidades de la enseñanza que veremos a continuación 
ayudarán al profesor a descubrir métodos, formas y procedimientos adecuados para una eficiente didáctica de la filosofía.

C) Modalidades de la enseñanza

La actividad estimulante que el maestro ejerce sobre el alumno no puede ser arbitraria o caprichosa. El acto didáctico es de tal complejidad que exige una serie de recursos que aseguren el aprendizaje por parte del alumno.

Entre los varios recursos didácticos nos detendremos ahora en el estudio de los métodos, procedimientos y modos en la enseñanza.

\section{El método didáctico}

Método es el camino, idealmente mejor, que conduce al fin de la didáctica. Decir "método" es decir "orden": es el orden que seguimos para evitar errores y encontrar la verdad (Balmes).

La formulación del método depende de dos determinaciones:

- de la naturaleza del CONTENIDO del saber y, en consecuencia, de exigencias lógicas o conceptuales, y

- de la naturaleza del PROCESO del aprendizaje, es decir, de exigencias sicológicas.

Estos dos aspectos son inseparables: condiciones objetivas (de la materia) y subjetivas (del alumno).

En la historia de la metodología didáctica se pueden encontrar dos grandes categorías de métodos: los clásicos y los modernos.

Los clásicos (típicamente lógicos) ven en el proceso de aprendizaje una función estrictamente intelectual; los modernos (de la escuela activa) se fundan en una 
complejidad de funciones (cognoscitivas, afectivas, apetitivas, motrices).

- Métodos lógicos (= clásicos)

Son los que se apoyan en las funciones mentales esenciales al proceso cognoscitivo. En este sentido, el movimiento del pensamiento se desenvuelve fundamentalmente en cuatro formas:

- inducción: procede de los hechos a la formulación de las leyes; de lo concreto a lo abstracto; de lo particular a lo general.

- deducción: desciende los principios a los hechos; de lo abstracto a lo concreto; de lo general a lo particular.

- análisis: procede por descomposición o separación de los elementos.

- síntesis: recompone los elementos simples en la totalidad.

- Métodos sicológicos (= modernos)

Son los que ponen en juego toda la actividad del alumno, y no solamente el entendimiento racional. La metodología moderna, sin excluir los métodos lógicos, ofrece una gran variedad de técnicas y procedimientos: método de proyectos, de centros de interés, de contrato, de discusión, de investigación activa y colectiva, etc.

Son métodos que, destinados a estimular al máximo la autoactividad del alumno, exige todo el empeño de la responsabilidad y actividad del profesor.

Los métodos se concretan en una variedad de procedimientos y de modos en la enseñanza, que constituyen precisamente la realidad didáctica en acto. 
2. El procedimiento didáctico

Es la aplicación particular de un método. Su clasificación depende de los métodos didácticos.

- Procedimientos inductivos

- observación: consiste en promover, provocar, proyectar, intencional y activamente, la atención sobre las cosas y los hechos, tal como se presentan espontáneamente al sujeto.

- experimentación: es una observación previamente preparada, en relación a un fenómeno, tomado como objeto de estudio, en determinadas condiciones.

- comparación: es la consideración sucesiva o simultánea de dos o más objetos de conocimiento, con el fin de establecer semejanzas o diferencias.

- abstracción: consiste en proyectar la atención sobre un determinado elemento de un todo, con exclusión de los otros componentes que pertenecen al mismo todo.

- generalización: consiste en extender los resultados de estudio de un cierto número de objetos particulares, a todos los de la misma especie, género o clase.

- Procedimientos deductivos

- aplicación: es una forma más simple, es la acción de referir un principio general a uno o más casos particulares.

- comprobación: consiste en cotejar una realidad con otra; puede ser inmediata (mediante una rápida observación o cálculo mental) 
o metódica (mediante una revisión esmerada y metódica, o una prueba de operaciones)

- demostración: procedimiento estrictamente lógico que pretende alcanzar la certeza de ciertas conclusiones.

- Procedimientos analíticos

- división: es un acto intelectual que realiza la mente cuando advierte que no puede abarcar la totalidad de un objeto dado.

clasificación: es el ordenamiento de una agrupación (individualidades objetivas o conceptos particulares) en base a analogías más o menos precisas.

- Procedimientos sintéticos

- conclusión: es la fase Terminal y definitiva de un análisis, o del desarrollo de una hipótesis formulada.

- definición: es la explicación exacta, clara y breve de una cosa.

- resumen: es la unificación orgánica de los elementos esenciales desarrollados a lo largo de una temática.

- recapitulación: consiste en recordar sumariamente lo estudiado, didácticamente debe ser completa, articulada e integrada por representaciones gráficas; puede ser parcial o total.

\section{Los modos de enseñanza}

Se trata de diversas modalidades de intercambio en el acto de la enseñanza-aprendizaje, desde el punto de vista de la relación "alumno-profesor”. 
Fundamentalmente existen cuatro modos:

- El modo individual

Este modo se da cuando la relación didáctica tiene lugar entre el profesor y un alumno particular. Es posible y muy útil en la instrucción privada; se hace prácticamente imposible en la enseñanza colectiva.

- El modo mutuo

Este modo se da cuando se cuenta con la colaboración de alumnos más adelantados en la enseñanza colectiva: los alumnos se instruyen recíprocamente. Tienen ventajas y desventajas: la cercanía sicológica y la inmadurez científica.

- El modo simultáneo

El profesor dirige su explicación a todos los alumnos simultáneamente. Es el modo colectivo que generalmente se usa en todas las escuelas.

- El modo mixto

Es el que se beneficia de los tres modos precedentes, combinados de diversas maneras.

D) La lección

La lección constituye el punto céntrico de la didaxis; estudiar su estructura es considerar todos los elementos del acto de enseñar. Debe distinguirse entre lección "verbalista" y lección "verbal". La primera absorbe toda la enseñanza; la segunda, estimula e integra la actividad personal del alumno.

Partiendo de la lección tradicional a la lección activa, podemos distinguir cuatro tipos de lección:

1) lección LOGO-CÉNTRICA 
a. Principio: dar el máximo de contenido, al mayor número de alumnos, en el menor tiempo posible.

b. Características:

Método $=$ deductivo

Procedimiento $=$ demostración

Modo $=$ simultáneo

Actor principal $=$ profesor

Centro de interés $=$ el programa

c. Fases: 1. Explicación: el profesor propone el tema y lo desarrolla.

2. Estudio individual: el estudio lo realizan los alumnos por su cuenta; a veces guiado por el profesor.

3. Recitación: toma la lección. No necesariamente al pie de la letra; puede servir de ampliación o ulterior explicación. Puede llevarse a cabo con discusión de grupos, con diálogos.

2) lección SICO-CÉNTRICA

d. Principio: unificar la materia concentrándola de acuerdo al desarrollo e interés del alumno.

e. Características:

Método $=$ inductivo

Procedimiento $=$ observación

$$
\text { Modo }=\text { mixto }
$$

Actor principal $=$ Profesor + alumno

Centro de interés $=$ el alumno

f. Fases 
1a forma: 1. Observación: (intuición). Examen del objeto que se estudia.

2. Asociación: (organización). Elaboración de los datos clasificándolos por categorías.

3. Expresión: exposición de lo aprendido

concreta: dibujo, composición, modelaje, construcción

abstracta: vocabulario, fórmulas

2a forma: 1. Introducción: consiste en poner en contacto con el tema, dando ideas fundamentales, modos de desarrollarlo, fuentes de consulta (= síncresis)

2. Ejecución: es el trabajo concreto. Puede hacerse por grupos, con diálogo profesor-alumno, por trabajo individual, por bibliografía, etc. (= análisis).

3. Valoración: datos fundamentales, clarificaciones, definiciones, sistematizaciones, etc. (= síntesis)

3) lección EMPIRIO-CÉNTRICA

i. Principio: se basa en la experiencia del alumno.

j. Características:

La clase $=$ ambiente de experiencias y actividades La lección $=$ nace de la experiencia y de la búsqueda

Actor principal $=$ el alumno

Centro de interés = el alumno "en" experiencia.

k. Fases: 
1. Planificación: se propone el tema en forma democrática, de acuerdo a estos puntos:

- ¿el tema satisface nuestras necesidades?

- ¿el tema es práctico y posible realizarlo?

- ¿el tema interesa a la mayoría de los alumnos?

2. Desarrollo: consiste en la búsqueda de datos: visitas, entrevistas, bibliografía, experimentos, discusión, etc.

El profesor tiene la difícil misión de organizar y dirigir estas actividades. Debe ser "motivador y orientador".

3. Comunicación y valoración: los resultados parciales se ponen en común y se formulan juicios, actitudes, definiciones, clasificaciones, conclusiones.

4) lección INTEGRAL

1. Principio: síntesis de exigencias lógicas y sicológicas

m. Características: Tiene en cuenta:

- la estructura fundamental del proceso cognoscitivo

- los intereses de los alumnos

- la participación activa y experimental de los alumnos.

n. Fases: 1. Orientación inicial: de distintas formas el profesor pone al alumno en contacto con el tema de la lección.

2. Búsqueda y análisis: es la fase 
más activa de la lección.

3. Síntesis final: consiste en formar un cuerpo doctrinal, organizado en sentido lógico, de todo el material buscado y analizado por los alumnos, que se refieren al tema de la lección.

La lección integral ya no se puede reducir a una hora de clase, sino que toma proporciones amplias en cuanto al tiempo. Organizada de esta manera, la lección permite a los alumnos adquirir una experiencia de vida y una cultura más profunda que en las lecciones meramente expositivas (tradicional).

Este tipo de lección es "completo" en el sentido que dinamiza todas las facultades del alumno; pero es "incompleto" en cuanto que deja abiertas las puertas para un saber ulterior.

\section{PRINCIPIOS BÁSICOS DE LA DIDÁCTICA GENERAL}

1. Terminología

2. Factores de la enseñanza

A) Sujetivo-sicológicos
o. globalización
p. motivación
q. autoactividad

B) Sujetivo-personales

r. personalidad del profesor

s. sicología del alumno
3. Modalidades de la enseñanza

A) El método didáctico
t. métodos lógicos

u. métodos sicológicos

B) El procedimiento didáctico
v. inductivo
w. deductivo
$\mathrm{x}$. analítico
y. sintético 

z. individual
aa. mutuo
bb. simultáneo
cc. mixto

dd. logocéntrica

ee. sicocéntrica

ff. empiriocéntrica

gg. integral

\section{SEGUNDA PARTE: PROCEDIMIENTOS CON CRETOS EN LA DIDÁCTICA DE LA FILOSOFÍA}

La metodología de la enseñanza de la filosofía debe ser una combinación y una articulación de los diversos métodos, procedimientos y modos de enseñanza, en la que deben estar comprometidos tanto el profesor como los alumnos.

La eficiencia de la enseñanza-aprendizaje (docente-discente), en filosofía, está decididamente condicionada por la variedad y la dinámica de las técnicas que se empleen y por la constante interacción profesor-alumnos.

A continuación, presentamos una serie de posibilidades y de esquemas para poner la clase de filosofía en proceso de "enseñanza-aprendizaje".

\section{Exposición filosófico-didáctica}

- Conocida como "clase magistral" (también como "logocéntrica") consiste en presentar un tema de manera verbal. A pesar de las críticas que se hace a este sistema y al abuso ("verborrea") de algunos profesores, 
con todo, tiene su importancia metodológica, siempre que fomente el interés de los alumnos.

Para esto, la exposición filosófico-didáctica debe tener ciertas cualidades:

- precisión en el planteo de la cuestión filosófica,

- división de la materia en tantas partes como fuere necesario para su comprensión,

- ordenación lógica en la secuencia de la explicación.

Y desde el punto de vista didáctico-sicológico, se exige:

- elección de temas interesantes y motivantes,

- ejemplificaciones adecuadas,

- aplicación de las conclusiones a la experiencia de los alumnos.

- Dentro de la exposición didáctica está el planteamiento y el análisis; es tarea importantísima y no fácil. En función de la formación intelectual de los alumnos, es tan importante como el resto de la lección.

La exactitud y claridad del planteamiento y del análisis es de tan gran valor que puede considerarse como "un todo en sí mismo", y no solamente como parte de la estructura de la lección expositiva. En otras palabras, la lección expositiva pudiera consistir únicamente en el planteo y análisis de un problema, sin que sea necesario exponer y fundamentar su solución.

De otra parte, debemos afirmar que no son exposición filosófico-didáctica la mera descripción empírica, la narración y explicación de hechos, ni el discurso persuasivo.

La exposición filosófica es la que remite todas las cuestiones a su punto de origen intelectual, a sus principios últimos; es la que problematiza radicalmente toda afirmación de hecho, toda suposición de relaciones, toda 
atribución de valores y las lleva a sus últimas consecuencias.

- Presentamos en forma indicativa ejemplos de exposición filosófico-didáctica.

- La exposición puede consistir en la presentación de una tesis filosófica con el análisis crítico y la fundamentación correspondiente. Esta tesis puede ser suscrita por el mismo profesor, o la de un filósofo o escuela significativa histórica.

Lo importante es la familiarización de los alumnos con la estructura conceptual, con el meollo racional del enfoque.

Por ej.: "La intuición emocional de los valores; aspectos sicológicos y ontológicos".

- La exposición también puede ser la formulación de las grandes líneas de una doctrina o sistema filosófico. A más de informar a los alumnos, este método busca ofrecerles una suficiente penetración de temas y problemas, orientando la meta a un esfuerzo mental provocado por los planteos, dificultades y soluciones propios de dicha doctrina.

Por ej.: "El materialismo dialéctico".

- La exposición también puede consistir en presentar el estado actual de una cuestión filosófica, sintetizando las opiniones y las conclusiones sobre ella, de acuerdo al pensamiento de hoy.

Es deseable que el profesor permita a sus alumnos la interrogación y la observación polémica sobre sus planteos, dando así lugar al debate.

Por ej.: "Valoración filosófica del evolucionismo".

Estas formas indicadas no son las únicas, ni se excluyen recíprocamente; más aún, pueden integrarse. El 
carácter filosófico de estas formas de exposición depende del grado de la preocupación crítica durante la lección.

Debemos decir que "no son exposición filosófica” la descripción empírica, la narración de acontecimientos, la explicación de hechos, ni el discurso persuasivo.

Concretamente, la exposición filosófica es la que remite todas las cuestiones a su punto de origen intelectual; es la que problematiza radicalmente toda afirmación de hecho, toda suposición de relaciones, toda atribución de valores y los lleva a sus últimas consecuencias.

\section{Interrogación didáctica en filosofía}

- Es un recurso que facilita a los alumnos el estudio, la comprensión y la elaboración de determinado asunto; es decir, facilita el aprendizaje. Ahora nos referimos a la pregunta que se práctica en el transcurso de la lección. No tanto como control del saber, sino más bien como método de ulterior investigación, de descubrimiento y de profundización de un determinado tema. Esta interrogación didáctica ha sido criticada por la Escuela Nueva Activa por "atentar contra la espontaneidad de los alumnos". Otros didactas la consideran como "corona de la habilidad magistral y punto culminante de la vida docente" (Diesterweg), y "como el arte de las artes y la forma más didáctica de enseñar" (Ruiz Amado).

La pregunta didáctica complementa, refuerza y aviva la atención y la reflexión, estimulando la elaboración mental, dirigiendo la misma atención y la participación de los alumnos en el aprendizaje.

De otra parte, la pregunta no es prerrogativa del profesor; también el alumno puede y debe preguntar. De esta manera se crea un verdadero diálogo entre profesor y alumnos. 
- En la lección de filosofía la pregunta constituye un caso especial en razón de la estrecha relación que existe entre la enseñanza filosófica y el filosofar (capacidad de crítica y de reflexión creadora). No es una simple demanda de datos, sino una indagación encaminada a lo que es universal y originario en el pensamiento y en el ser. Constituye una permanente motivación a la confirmación de todos los puntos de vista posibles. Toda pregunta filosófica, propiamente dicha, debe guiar la mente de los alumnos a una investigación metafísica.

- Dejando de lado algunos tipos de preguntas, nos detenemos a las que interesan en la didáctica de la filosofía.

- de acuerdo a la amplitud de las respuestas:

- preguntas cerradas (=de respuesta limitada) son aquellas que demandan una respuesta breve y precisa. Son las llamadas "alternativas, polares o excluyentes" como por ej. las de "SÍ" o "NO", las de "verdadero" o "falso".

Cuestiones discriminatorias

Coloque sobre las líneas, frente a cada una de las proposiciones siguientes las letras $\mathrm{V}$ o $\mathrm{F}$ para indicar su verdad o falsedad:

1. El método didáctico en filosofía es totalmente independiente del método de conocimiento

2. El término "inducción” es sinónimo de "abstración"

3. El discurso persuasivo es exposición filosófica 
4. La pregunta efectiva supone

el deseo de alcanzar un

contenido cognoscitivo

5. Toda pregunta filosófica

debe tender a una

investigación científica

- preguntas abiertas son las que dan lugar a que los alumnos formulen libre y ampliamente sus propias opiniones sobre el asunto tratado.

Por ej.:

¿Cómo explica Ud. la libertad moral en un mundo determinado?

- de acuerdo a la especificidad de las preguntas:

- preguntas lingüísticas son las que se refieren a términos o expresiones del lenguaje.

Por ej.:

¿Qué significa...? ¿Cómo se usa este término...? ¿Cuál es el análisis concreto de esta expresión...?

- preguntas lógicas son las que implican un razonamiento.

Por ej.:

Esta conclusión ¿es causa o consecuencia? Este argumento ¿es apropiado al caso en cuestión?

- preguntas epistemológicas y críticas son las gnoseológicas, que atañen a cuestiones epistemológicas o criticas.

Por ej.:

¿Cuál es el fundamento de la validez de un silogismo? 
¿Cómo es posible una causa no causada?

- preguntas ontológicas son las que se refieren a problemas metafísicos.

Por ej.:

¿Por qué existo? ¿Cuál es el fin del hombre?

Existe también la pregunta diferida: es la que el profesor plantea, dejándola como tarea a los alumnos.

En este caso, la respuesta debe ser más detallada y más razonada; por eso algunos profesores la exigen por escrito.

- Condiciones de una pregunta didáctica:

- clara: libre de ambigüedades, de doble sentido u oscuridad

- correcta: expresada en lenguaje sintáctico y vocabulario adecuado

- sencilla: libre de complicación y de fácil captación

- breve: la formulación debe ser hecha con pocas palabras.

\section{Método dialogado (= socrático)}

- Se da este método cuando la dirección del aprendizaje se desarrolla en un encadenamiento de interrogaciones y respuestas, incorporándose así el pensamiento de los alumnos en el proceso dialéctico de la reflexión filosófica.

El fundamento de este método se halla en la misma naturaleza del "filosofar", que se nutre del intercambio y de la oposición de las conciencias reflexivas. Lo característico de la clase dialogada es el desarrollo del tema a base de preguntas y respues- 
tas con la participación del profesor (participación directa o indirecta).

- Procedimiento general

No puede indicarse un modo fijo, determinado y único para el diálogo en la clase; con todo, podemos dar una orientación de base.

- Como iniciación del diálogo debe plantearse una temática apta para despertar el interés de los alumnos; temática densa de contenido reflexivo, de inquietudes y al nivel intelectual del alumnado.

- El punto de partida debe ser lo más asequible y sencillo posible, a fin de crear un clima de autoconfianza en los alumnos, facilitando así su participación.

- Se deberá partir de las cosas comunes de la vida diaria, para llegar a los enfoques propiamente filosóficos.

- Cuando algo "no va” (desarticulación, dificultades sin aparente solución, cansancio, pérdida de interés, etc.), el profesor debe estar listo para intervenir (siempre en forma dialógica) con interrogaciones de auxilio.

Por ej.:

¿En cuántos sentidos puede usarse este término?

¿Los hechos aducidos corresponden con la afirmación dada?

¿Qué se requiere para que " $\mathrm{X}$ " sea cierto (o falso)?

¿El problema planteado es un problema de hechos, de terminología o de valores?, etc. 
- Esquema sugerido para el desarrollo de una clase dialogada.

1. Presentación del tema y de los problemas colaterales implicados

2. Análisis de respuestas, hipótesis explicativas, posibles soluciones.

3. Argumentación en relación a las alternativas planteadas y a sus consecuencias.

4. Recapitulación o conclusión: comparación de las distintas conclusiones y elección eventual de la más verosímil ya sea por su claridad, seguridad o fecundidad.

Requisitos de aplicación.

- Clase poco numerosa. Un grupo de 10 a 15 alumnos es un número ideal para una conversación socrática.

- Disponibilidad de tiempo para que el diálogo pueda desenvolverse adecuadamente. Las sesiones de dos horas de clases sucesivas resultan muy convenientes para el caso.

- Un profesor con buen entrenamiento dialéctico, dominio suficiente de los medios de expresión, agilidad de intelección, autoridad magisterial y poder de incitación. Con gran capacidad de receptar los aportes de los alumnos ya para aceptarlos, rectificarlos o reemplazarlos cuando fuere necesario.

- Alumnos con suficiente base de cultura filosófica, con agilidad y madurez intelectual. De lo contrario el diálogo resultaría forzado o termina en un monólogo del profesor.

\section{Consideraciones finales}

Debe evitarse el forzar la solución de los problemas planteados. La carencia de respuestas satisfactorias al 
final de la clase, no significa necesariamente que el diálogo socrático haya fracasado. En filosofía, una dificultad o controversia no resuelta pero bien analizada, es tan fecunda como una solución definitiva. Didácticamente, lo importante es que el diálogo se haya conducido con método y rigor, y que las cuestiones queden debidamente precisadas y formuladas.

El profesor debe evitar caer en la tentación de un socratismo fácil y superficial: la de emplear el arte de la persuasión en este tipo de diálogo.

El profesor no debe manejar su saber y su retórica para "persuadir" a los alumnos hacia determinada solución.

Es importante no usar la "ironía" en las intervenciones de los alumnos en clase. Este comportamiento hiere los sentimientos de los alumnos (particularmente de los jóvenes) y los inhibe de participar en el diálogo. El método pedagógico actual renuncia al socratismo histórico.

\section{Método fenomenológico-crítico}

En realidad son dos métodos que se distinguen suficientemente el uno del otro, pero que pueden fusionarse y combinarse en una misma metodología.

- El filosofar fenomenológico consiste en la descripción de la conciencia, con exclusión de toda idea previa, teoría o tesis interpretativa; con exclusión de todo supuesto sobre la existencia o no de aquello que se da.

- El filosofar crítico-trascendental consiste en preguntar por las condiciones de posibilidad de una determinada instancia, sea del conocimiento de la acción, sea de la naturaleza o de la conciencia.

- Aplicación metodológica 
Se pueden distinguir dos momentos de aplicación didáctica en la enseñanza-aprendizaje de la filosofía:

1) El momento fenomenológico

Este momento consiste en la descripción de datos, independientemente de toda consideración o actitud apreciativa o valorativa.

La atención se centra sobre el "fenómeno", en actitud de intuición "eidética" (=captación de la idea, de la esencia de lo dado, de lo visto).

Dicha intuición contempla "lo dado" (el fenómeno) haciendo abstracción de cualquier interpretación científica.

Esta didáctica fenomenológica puede presentar dos variantes:

- los ALUMNOS proceden al examen y descripción del fenómeno bajo el control del profesor;

- El PROFESOR realiza la descripción y los alumnos siguen este trabajo tomando apuntes y pidiendo aclaraciones o añadiendo observaciones.

El profesor deberá atenerse a aprehender sólo lo que "se da", en cuanto y como se da; no deberá recurrir a los conocimientos, convicciones, fórmulas o conceptos previos, a fin de evitar el caer en prejuicios (=los ídolos de Francis Bacon) que desvían a la conciencia en su ruta hacia la verdad. El profesor se ajustará al cumplimiento de esta prescripción y ejercitará al alumno en tal control.

2) El momento crítico

Una vez lograda la intuición de determinado fenómeno, se pasa al segundo momento: el crítico. El profesor motivará a sus alumnos mediante la formulación de la 
pregunta por las condiciones de posibilidad: “Cómo es esto posible?" ¿"No es paradójico que suceda?" ¿"Por qué esto y no aquello"? Estas preguntas introducen la inquietud crítica en los alumnos.

Se trata de hacer que los alumnos alcancen la intelección de la razón o fundamento de los hechos o de las cosas.

Pudiera suceder que al mismo tiempo que se alcanza una respuesta satisfactoria, se redefine el problema o se aclaran sus límites, aspecto este muy importante pues los alumnos van alcanzando un rigor crítico, propio de la enseñanza aprendizaje de la filosofía.

Este es el momento de los interrogantes, de la "elaboración crítica", de la búsqueda de respuestas, de la confrontación con otros fenómenos que interesan al hombre, en general.

La didáctica de este momento crítico puede presentar dos variantes:

- los ALUMNOS formulan cuestiones críticas pensadas "anticipadamente" o que surgen durante la clase;

- el PROFESOR formula los interrogantes para motivar la reflexión de los alumnos y para comprometerlos en su solución.

La formulación y el tratamiento de las preguntas críticas pueden hacerse en dos direcciones:

a. orientada por los datos de la experiencia moral: ¿cómo es posible la libertad y dentro de qué limites? ¿atendiendo a los hechos de la experiencia moral (el deber, la responsabilidad y la culpa) hasta dónde puede definirse? 
b. orientada por la necesidad de comprender la existencia de los actos libres no en forma aislada, sino como un "todo" de la realidad:

¿cómo deberá concebirse el mundo y cómo se inserta en él, el hombre libre?

Si la realidad mundana está determinada por causas y fines ¿es posible la acción humana libre?

\section{Métodos sicológicos}

Son los que tratan de "individualizar" la enseñanza mediante las dinámicas grupales. La clase es un ambiente social en la que cada uno (profesor y alumnos) tiene su puesto y sus funciones: el proceso enseñanzaaprendizaje debe hacerse con la colaboración de todos y de cada uno.

Las dinámicas de grupo en la didáctica, no solamente obvia la dificultad de trabajar con grupos numerosos, sino que permite la eficiencia de la enseñanza escolar. El cambio de estructura de la clase, de la "vertical" a la "horizontal", de la del gran grupo al pequeño grupo, de la clase "tradicional" a la clase "con dinámicas" es muy ventajoso tanto para el profesor como para los alumnos.

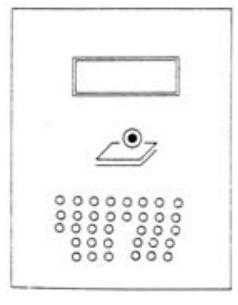

Clase tradicional

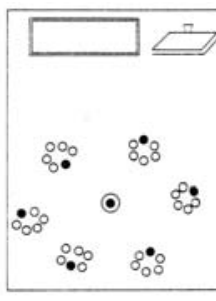

Clase con "dinámica"

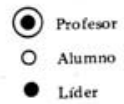

Nota: dinámica de grupos. Esta expresión comenzó a circular por el año 1935, y fue consagrada en los EE.UU. por Kurt Lewin en 1944. 


\section{- Ejemplos de esta metodología}

\section{1) El Phillips 66}

Esta dinámica consiste en un intercambio de ideas, en grupos de 6 personas, durante 6 minutos, sobre un tema señalado por el profesor. Uno de los miembros de cada grupo hace las veces de moderador (líder) y de relator, para presentarlo a toda la clase.

Para formar los grupos basta con que tres personas de la primera fila se den la vuelta y dialoguen con las otras tres de la segunda fila, sin necesidad de levantarse todas. En la exposición se puede hacer uso del pizarrón.

\section{2) El cuchicheo}

Esta técnica consiste en un diálogo, más o menos breve, entre parejas o en grupos pequeños. Se le ha dado este nombre a causa del murmullo sordo que produce al hablar en voz baja. Algunos la llaman "runruneo".

Esta técnica se puede emplear cuando el profesor pide una respuesta rápida sobre un asunto o una opinión, en que quiere la participación de todos.

\section{3) La lluvia de ideas}

Algunos la llaman "tormenta cerebral" (brainstorming). Esta técnica tiene por objeto la producción de nuevas ideas, de estimular la imaginación creadora.

Las condiciones que se requieren para esta dinámica son:

- debe tratarse de un problema filosófico "real";

- el problema debe ser "simple". Si es complejo, deberá descomponerse en partes simples.

- el problema deberá quedar "claro" ante los alumnos. 
Las tres fases de esta metodología dinámica:

a. Exposición preliminar del problema

La hace el profesor. Esta exposición debe ser bien preparada, para que los alumnos la entiendan con toda claridad.

b. Tormenta de ideas

- los alumnos lanzan ideas, por turno. Una vez que el primero dice algo, el siguiente se esfuerza por "seguir - la corriente" y agregar algo (una aprobación, un rechazo, una objeción).

- el profesor no interviene en la producción de ideas; su actitud personal es de acogida y no-directiva.

- el profesor actúa animando, estimulando; no criticando ni desaprobando.

- los mismos alumnos deben evitar el criticar, para dejar libre la imaginación y la creatividad.

- es más importante la "cantidad" de ideas, que la "calidad".

c. Depuración y crítica de las ideas expuestas

Es el momento de la crítica filosófica, de la sistematización, de las clarificaciones.

En esta fase ya no se busca la cantidad, sino la "calidad" de lo expuesto por los alumnos.

4) El estudio de casos

Esta técnica consiste en el planteamiento de un problema, la investigación de sus causas y la búsqueda de posibles soluciones al mismo.

El objetivo de esta dinámica es la de estimular el potencial analítico de los participantes en ella, su espíritu de iniciativa y su capacidad para tratar de resolver situaciones humanas complicadas. 
Las fases de esta dinámica pueden ser:

a. Presentación del caso

Un caso bien presentado debe tener las siguientes cualidades:

- Presentar una situación concreta (= autenticidad del caso)

- Una situación-problema que exige un diagnóstico y una solución.

- Presentar la situación en "su totalidad"

- Que se refiera a una temática filosófico-existencial.

b. Búsqueda de las causas

La participación de los alumnos puede hacerse a través del Phillips 66, o la lluvia de ideas. El profesor será nodirectivo "en el fondo": no da sugerencias, no indica posibles causas, no evalúa las respuestas de los alumnos. Refleja sistemáticamente las intervenciones de los alumnos.

El profesor será directivo "en la conducción": da la palabra, da igualdad de oportunidades a todos los alumnos, cronometra síntesis parciales.

Se hace un listado de las causas sugeridas y luego se hace el análisis de las más significativas.

c. Estudio de posibles soluciones

Esta es la fase "reflexiva" y crítica.

La reflexión se puede orientar en 4 direcciones:

- mirada hacia atrás: el contexto y las causas del problema

- mirada hacia arriba: los principios filosóficos que entran en la solución del caso, o que brotan del problema

- mirada al rededor: descubrir situaciones análogas al caso

- mirada adelante: es el aprendizaje para aplicar a otros casos. 
5) Mesa redonda

A veces conviene presentar un tema desde diversos puntos de vista contradictorios. Por ej. las distintas posiciones filosóficas sobre la libertad: fatalismo, determinismo, indeterminismo.

Los que van a exponer el tema pueden ser los mismos alumnos convenientemente preparados.

- El profesor se pone de acuerdo en el modo a seguir, sobre el orden en que hablarán y el tiempo que emplearán en la exposición individual.

- El profesor se sentará en el centro, y a su lado los contraversistas alumnos.

- El profesor presentará el tema brevemente; luego irá dando la palabra durante 10 minutos a los expositores, procurando que se turnen las distintas posiciones.

- El profesor sintetiza los diversos puntos de vista expuestos, y dará oportunidad a una segunda vuelta para que se aclaren los puntos que quedaron oscuros.

Esta dinámica puede emplearse en temas "controvertidos”. Parece sumamente útil para aclarar posiciones, para inquietar a los alumnos por diferentes doctrinas, o para profundizar una temática determinada.

Se debe cuidar de que no se convierta en una polémica sin fin y estéril.

6) La entrevista colectiva

Esta dinámica pretende aprovechar hasta el máximo la ciencia de una persona experta en algún tema filosófico (sistemático o histórico), pero siguiendo los intereses de los alumnos. 
En lugar de que el experto dé una conferencia, el profesor selecciona unos pocos alumnos para hacer una serie de preguntas al experto.

El perito debe saber globalmente el tema, pero sin saber en concreto cuáles serán las preguntas por parte de los alumnos preparados para el caso.

- El profesor presentará primero a los alumnos interrogadores, luego el tema en general, y finalmente al entrevistado.

- El entrevistado hará una exposición clara y breve sobre la temática.

- Luego se da comienzo al interrogatorio, de acuerdo al orden establecido y en forma considerada y cordial.

- Al término de esta entrevista el profesor resumirá el contenido, y abrirá el Plenario al curso.

Para que esta dinámica pueda alcanzar su objetivo, el tema deberá ser del interés de los alumnos, y el entrevistado deberá tener la cualidad de una comunicativa interesante y ágil, sin descuidar la profundidad y seriedad del caso.

\section{Método de ejercicios escritos}

Los estudios filosóficos en la educación media deben tener un tiempo y un espacio para la "expresión escrita”. Este es un aspecto al que conviene ejercitar a los alumnos.

Es recomendable destinar periódicamente durante el curso, el tiempo necesario a este método, incluso para dejar constancia escrita del progreso filosófico. 
- Posibilidades y formas

- la toma de notas: esta es la forma más simple y espontánea de las formas escritas: tomar nota de lo que el profesor expone. Lo que se debe consignar no es "la letra", sino "las ideas" principales. Es bueno que, para el caso, dispongan de un cuaderno o libreta de apuntes.

- el comentario escrito: es como un comentario oral, pero elaborado por escrito y siguiendo las normas de redacción. Normalmente el comentario escrito es más largo y más trabajado que el oral. Como comentario, no requiere toda una sistematización como una pequeña disertación escrita.

- el resumen: es una forma sintética de presentar las ideas y pensamientos principales de un texto. No es un comentario ni una interpretación o apreciación personal de dicho texto. El resumen se hace reduciendo el texto en cuestión (normalmente a un tercio) con las propias ideas principales y conservando, incluso, las mismas expresiones del autor.

- el ensayo: es una forma breve de escrito, y se caracteriza tanto por la libertad en la elección de temas, como por la manera y estilo de su presentación. Es un modo muy apropiado de "filosofar con la pluma". Este tipo de trabajo puede tomar las formas de "pensamientos, máximas, artículo periodístico" que, eventualmente, podría servir de material para revistas escolares o para el periódico del establecimiento o del curso. 
7. El método de lectura y comentario de textos

\section{filosóficos}

Este método es absolutamente necesario para motivar un auténtico filosofar.

La lectura frecuente y sistemática de obras filosóficas y su correspondiente comentario es un ejercicio que compromete seriamente al profesor y a los alumnos.

- Finalidad de este método

- analizar y comprender el pensamiento de determinado autor;

- identificar las características de una época, escuela o corriente filosófica, y reconocer sus valores;

- enriquecer los conocimientos filosóficos y alimentar el deseo al filosofar;

- capacitarse para escribir ensayos, disertaciones u otros trabajos de carácter filosófico.

1) Lectura de textos

a. Qué puede leerse:

Siendo tan vasta la producción filosófica, es preciso que el profesor selecciones algunas obras o fragmentos significativos para determinados temas.

- según el acontecimiento teórico

Conviene trabajar sobre obras específicamente filosóficas, y no sobre obras de tipo literario, científico o moral; aunque algunas obras filosóficas entrelazan la literatura con al filosofía²

- según el grado de dificultad

Tratándose de alumnos de secundaria, conviene elegir textos propios para esta edad. Para esto conviene tener en cuenta: 
- que el texto sea unitario, simple y no de una complicada articulación conceptual;

- que no presuponga demasiada información previa;

- que el lenguaje sea directo y claro;

- que el texto sea rico en análisis de conceptos, de conclusiones, de críticas reflexivas para el filosofar de los alumnos.

- según la estructura de la obra

De acuerdo con la extensión, la integridad o la fragmentación del texto:

- lectura de obras completas de no mucha extensión

- lectura de un artículo o ensayo breve

- lectura de trozos escogidos de diferentes filósofos, de distintas épocas, escuelas o nacionalidades, o de diferentes temas.

b. Cómo puede leerse

Hay dos maneras o procedimiento de lectura que se conocen como lectura "horizontal" y lectura "vertical".

- la lectura horizontal

Es una lectura rápida, superficial (pero completa) de la obra; una información general y global. No supone un análisis, pero da al alumno una visión de conjunto. Generalmente se da como labor fuera del aula.

- la lectura vertical Es una lectura pausada, profunda, con la voluntad de penetrar en el texto; esta lectura es de tipo analítico e interpretativo. 
Frente a la lectura de un texto filosófico, el alumno puede tomar dos actitudes opuestas entre si:

- Una actitud objetiva, impersonal y dogmática. Se le da crédito al filósofo: el alumno se somete a su pensamiento, coincide con sus afirmaciones y negaciones, se identifica con el escritor.

- Una actitud sujetiva, llamada por Ricoeur "escéptica"; es decir, el alumno se enfrenta con el autor y el texto. Se trata de confrontar el texto con las propias ideas y reflexiones, y de rebatirlo o negarlo si se resiste a la asimilación.

2) Comentario de textos

Este momento es el más importante; es una verdadera actividad de hermenéutica filosófica (= interpretación, explicación, deducción...). Entre leer y comentar existe la misma diferencia entre contemplar un regalo de navidad envuelto, y el abrirlo y contemplarlo directamente. El comentario es el momento "deductivo" de la lectura de un texto. Una lectura sin su correspondiente comentario, sin interpretación, sin penetración puede resultar interesante, pero no conduce al filosofar crítico.

\section{- Preguntas-guías}

- ¿Qué dice el autor, cómo lo dice y qué quiere decir?

- ¿Qué no dice el autor?

- ¿Por qué y para qué lo dice?

- ¿En qué circunstancias lo dice?

- ¿Debe decirlo? ¿Está bien dicho? ¿Qué razones tiene para decirlo? 
- ¿Quién hace el comentario?

- El profesor: a manera de modelo, oralmente consignando en el pizarrón los pasos o puntos esenciales del comentario.

- Los alumnos: a manera de imitación, por escrito primero para la corrección del profesor o para la exposición en clase. El comentario nunca es una improvisación, sino un trabajo serio que pide tiempo y constancia.

- Los pasos de un comentario

- En cuanto a los términos:

- identificación de términos poco usuales o difíciles

- búsqueda del significado de dichos términos (diccionario filosófico)

- En cuanto a las ideas expresadas en el texto:

- selección de las ideas; distinguir las ideas principales de las secundarias

- estructuración de las ideas principales (identificarlas)

- descubrimiento de la idea central; es decir, los que se podría escribir como título del comentario.

- En cuanto al autor:

- ubicación de su vida (año, lugar)

- orientación filosófica de su pensamiento

- obras escritas por él e importancia de su pensamiento. 
3. El método audiovisual

En menor medida que en otras disciplinas de estudio, la enseñanza-aprendizaje de la filosofía está haciendo uso de los medios de comunicación social, como las grabaciones, la radio, la televisión y el cine.

- Como aplicación sistemática de grabaciones y emisiones radiales pueden mencionarse los discos con textos leídos de filósofos de renombre (Heidegger, Sartre, etc.), y los ciclos sobre filosofía contemporánea y coloquios entre personalidades en filosofía organizados por la $\mathrm{BBC}$ de Londres.

- Mucho más elaborados son los programas de televisión escolar, como los de Francia en las emisoras del Estado. Son programas de duración media de 30 minutos, están a cargo de profesores universitarios y de educación secundaria, quienes discuten sobre un determinado tema (eventualmente explican y comentan textos filosóficos).

Cada emisión es acompañada de un texto que contiene una recapitulación de las cuestiones, una breve guía bibliográfica y un glosario de los términos técnicos empleados.

- Por lo que toca a cine enseñanza-aprendizaje, existen algunas películas dedicadas a la enseñanza de la filosofía, como las editadas en inglés por la enciclopedia Británica con la participación de pensadores, científicos y hombres públicos significativos de nuestros días (Russell, Nehru, Wrigth...) Estos pensadores son interrogados sobre sus ideas; a veces se desarrollan temas como: el bien y el mal, la justicia, la libertad, Dios, etc. 
Si bien estos medios didácticos auxiliares no pueden reemplazar la enseñanza magisterial que hemos presentado, con todo adquieren una importancia muy grande como medio para ampliar los conocimientos adquiridos en clase, o como suplencia ante el déficit de personal docente especializado.

\section{TERCERA PARTE: PROCESO DE ENSEÑANZA Y EVALUACIÓN}

Toda enseñanza aprendizaje se realiza dentro de un proceso integral que abarca:

- el programa (diseño del contenido)

- el aprendizaje (realización del programa)

- la evaluación (valoración del aprendizaje)

En esta tercera parte de nuestros apuntes sobre enseñanza-aprendizaje de la filosofía, analizaremos brevemente la evaluación en su aspecto didáctico.

La evaluación escolar no debe limitarse solamente a una valoración sobre el rendimiento de los alumnos, sino que entra también en dicha evaluación el determinar en qué medida han sido efectivos el programa, la metodología empleada en la enseñanza y, en general, la eficiencia del profesor.

El planteamiento sobre la evaluación, en realidad, se basa en un principio sencillo; responde a estas dos preguntas:

- ¿qué es lo que se intenta evaluar?

- ¿qué medios se emplean para recoger los datos evaluativos? 


\section{Lo que se debe evaluar}

¿Qué es lo que se intenta medir? La respuesta a esta pregunta no resulta difícil: lo que se quiere saber es: hasta qué punto se han alcanzado los objetivos propuestos en esta asignatura y, como consecuencia, saber la eficiencia del programa y la operatividad de los métodos empleados por el profesor.

- De una parte, los objetivos de la enseñanza de la filosofía son precisos:

- potenciar el hábito mental de análisis y crítica personal;

- conocer las principales doctrinas filosóficas (el "corpus" fundamental del saber filosófico);

- poseer un sentido objetivo en el pensar, hablar y escribir, que proceda desde un punto de vista lógico y con rigor científico.

- Pero de otra parte, se hace un tanto difícil esta cuantificación:

- muchas de las veces la evaluación del profesor se fundamenta en una apreciación muy general de los datos aportados;

- otras veces es fruto de sujetivismo o prejuicios sobre determinado alumno;

- después de la evaluación queda como "flotando en el aire" una serie de interrogantes:

¿se han formulado las pruebas de una manera adecuada?

¿se tienen datos suficientemente significativos para analizar la consecución de los objetivos? ¿se puede alcanzar un porcentaje mayor de objetividad evaluativa? 
Sin pretender ofrecer el "optimum" de un proyecto de evaluación, presentamos un procedimiento no complicado y claro. ${ }^{3}$

Se trata de dos cuadros de doble entrada:

- En el eje vertical se formulan los "contenidos" del programa;

- en el eje horizontal aparecen los “objetivos" a alcanzar.

Cuadro 1.

\begin{tabular}{|c|c|c|c|c|}
\hline UEMIDAD & $\begin{array}{c}\text { Memorización } \\
\text { de hechos }\end{array}$ & $\begin{array}{c}\text { Formación de } \\
\text { conceptos }\end{array}$ & $\begin{array}{c}\text { Capacidad de } \\
\text { anál. síntes. }\end{array}$ & $\begin{array}{l}\text { Capacidad de } \\
\text { relac. crit. }\end{array}$ \\
\hline $\begin{array}{l}\text { Contexto } \\
\text { histórico } \\
\text { y autores }\end{array}$ & & & & \\
\hline $\begin{array}{l}\text { Temática } \\
\text { central }\end{array}$ & & & & \\
\hline $\begin{array}{l}\text { Planteamiento } \\
\text { ontológico }\end{array}$ & & & & \\
\hline $\begin{array}{l}\text { Planteamiento } \\
\text { antropológico }\end{array}$ & & & & \\
\hline $\begin{array}{l}\text { Planteamiento } \\
\text { ético-social }\end{array}$ & & & & \\
\hline
\end{tabular}


Cuadro 2.

\begin{tabular}{|c|c|c|c|c|}
\hline $\begin{array}{l}\text { OBJETIVOS } \\
\text { UNIDAD } \\
\text { TEMÁTICA }\end{array}$ & $\begin{array}{l}\text { Tiempo de } \\
\text { desarrollo }\end{array}$ & $\begin{array}{c}\text { Material a } \\
\text { usar }\end{array}$ & $\begin{array}{c}\text { Instrumentos } \\
\text { didácticos }\end{array}$ & $\begin{array}{l}\text { Medios de } \\
\text { motivación }\end{array}$ \\
\hline $\begin{array}{l}\text { Contexto } \\
\text { histórico } \\
\text { y autores }\end{array}$ & & & & \\
\hline Temática central & & & & \\
\hline $\begin{array}{l}\text { Planteamiento } \\
\text { ontológico }\end{array}$ & & & & \\
\hline $\begin{array}{l}\text { Planteamiento } \\
\text { antropológico }\end{array}$ & & & & \\
\hline $\begin{array}{l}\text { Planteamiento } \\
\text { ético-social }\end{array}$ & & & & \\
\hline
\end{tabular}

Aquí se proponen dos modelos de cuadros:

- el cuadro 1. = dirigido a los objetivos de los contenidos (programa)

- el cuadro 2. = dirigido a los objetivos metodológicos (operativos) 
Estos cuadros pueden formularse en dos niveles:

- programación general (global) de la asignatura: Lógica, Ética, Problemas filosóficos (de acuerdo a nuestro programa)

- programa específico de cada unidad.

Algunos profesores objetan que tales cuadros encuadra en forma demasiado rígida la enseñanza de la filosofía que, por naturaleza, no tiene la precisión de las materias exactas.

En realidad, no se trata de poner rigidez; lo que se pretende es eliminar la improvisación y el empirismo en una evaluación que quiere ser lo más objetiva posible.

2. Medios para obtener los datos evaluativos

Algunos profesores rechazan sistemáticamente las pruebas evaluativas, fundamentalmente por dos razones:

- Porque restringen la libertad del profesor para tratar el programa del modo que estime más adecuado (le resta tiempo para desarrollar el contenido).

- Porque pueden conducir al alumno a una desubicación de objetivos de la materia: estudiar en función de las pruebas.

Con todo lo que esto pudiera tener de verdad, lo cierto es que "las pruebas" evaluativas tienen gran importancia en la enseñanza-aprendizaje:

- No sólo sirven para evaluar el progreso de los alumnos, sino también para avaluar la propia tarea del profesor.

- Pasan a ser un acicate "externo" para el estudio, si bien no debiera ser la única motivación. 
- Son un instrumento didáctico fundamental: "No hay impresión sin expresión".

- Condiciones para la confiabilidad de las pruebas evaluativas:

- deben ajustarse a los objetivos formulados en la enseñanza de las materias filosóficas ${ }^{4}$;

- es mejor hacer varias pruebas cortas, que no una excesivamente larga;

- la formulación de las preguntas debe ser clara y precisa, sin posibilidad de equívocos;

- debe establecerse una valoración previa de cada uno de los datos que se intenta recoger de la prueba.

- Modelos de pruebas

Normalmente de han distinguido dos modelos básicos:

1. La prueba objetiva

Implica preguntas que están pensadas para dar lugar a una respuesta aceptable. La formulación de estas pruebas pueden ser muy variadas: completar una frase, discernir entre Verdadero y Falso, identificar la respuesta correcta, etc.

Estas pruebas objetivas son un modelo que se orienta principalmente a la memorización de hechos, de definiciones y de conceptos, pero puede usarse para evaluar el grado de comprensión de determinados contenidos y de relación entre ellos.

Se trata de una prueba ágil y de fácil aplicación; es laboriosa en su preparación, pero rápida para la corrección.

Es cierto que estas pruebas objetivas no son las mejores para evaluar la capacidad crítica y el pensar reflexi- 
vo filosófico, pero también es cierto que entre los objetivos de la enseñanza de la filosofía también se incluye una información sobre conceptos, autores y orientaciones filosóficas, para cuya medida estas pruebas objetivas son las más adecuadas.

\section{La prueba de ensayo}

Bajo estas pruebas se incluye un conjunto de ellas que permiten valorar niveles superiores de la actividad mental adquiridos por los alumnos, como son: capacidad de análisis, de síntesis, de reflexión, de crítica, de expresión, etc. Ya nos hemos referido a algunas de ellas como metodología de enseñanza: ejercicios para clasificar preguntas, estudio de casos, ejercicios escritos, lectura de textos, comentarios, ensayos, resúmenes, etc.

Estas pruebas llevan "mucho tiempo" en la ejecución por parte de los alumnos, y conlleva un gran "sujetivismo" en el juicio valorativo por parte del profesor. Justamente, para evitar este sujetivismo, existen algunos criterios y algunas técnicas:

- Al proponer un tema, éste debe formularse con preguntas precisas y bien determinadas.

- en lugar de enunciado general: "la responsabilidad moral",

- es mejor determinar más el aspecto pedido: "la moral, en relación con la libertad";

- y mejor aún si esta forma indicativa se hiciera en forma interrogativa: “ $¿$ La responsabilidad implica la libertad? ¿Por qué?”

- No debe reducirse la prueba a la escueta respuesta de una pregunta. Lo aconsejable es diversificar la 
respuesta a través de aclaraciones, ampliaciones, comparaciones, ejemplificaciones, explicaciones del porqué, oposiciones, etc. De esta forma el alumno puede manifestar el dominio o no de lo aprendido, su capacidad reflexiva y analítica.

- La temática propuesta debe contener problemáticas fundamentales y significativas sobre el saber filosófico; no debe detenerse en detalles sin importancia o en datos secundarios del programa.

- La prueba de ensayo "escrita" puede realizarse también con consulta de libros, apuntes u otros documentos. Esto es muy apropiado para una prueba filosófica, porque el alumno pone menos interés en la memorización, y más en el tratamiento reflexivo de los problemas.

- Con el fin de ampliar el margen de posibilidades de evaluar el rendimiento del alumno y darle la oportunidad de tratar aquellos asuntos que más le motiven, conviene proponer varios temas entre los cuales elegirá uno o más como materia de examen.

- Evaluación de la asignatura por parte de los alumnos Para muchos profesores existe todavía algún recelo a someterse a una evaluación por parte de los alumnos. El fundamento principal de este recelo se basa en la duda de que exista en ellos una suficiente formación que les capacite para realizar esta tarea.

La práctica de aplicación de cuestionarios a los alumnos, desmiente estas prevenciones, siempre que se guarden dos principios: 
- las respuestas deben ser anónimas;

- las cuestiones que se planteen deben incidir más sobre aspectos conductuales y didácticos que sobre contenidos.

Las preguntas que se formulan pudieran ser en base a los siguientes puntos:

- definición de los objetivos del curso;

- organización de la materia a dictarse y del curso;

- conocimiento de la materia por parte del profesor;

- variedad de técnicas en la enseñanza-aprendizaje;

- justicia en la calificación de los alumnos;

- atención especial en los trabajos de los alumnos;

- opinión general acerca del profesor;

- opinión general acerca del curso.

Cada una de estas preguntas es baremada por el alumno en una escala que va de 0 a 10.

\section{Notas}

1 Se considera a Edmund Husserl, filósofo alemán (1659-1938) como fundador de la fenomenología, método y sistema que afirma que la inteligencia humana puede intuir las esencias de las cosas de modo inmediato y cierto.

2 Por ejemplo: "Los pensamientos" de Pascal, "Así habló Zaratustra" de Niezstche, "El mito de Sísifo" de Camus, "El contrato social” de Rousseau, etc.

3 Sacado de V. SANTIUSTE, Didáctica de la Filosofía, Madrid, Ed. Narcea, 1984.

4 Ver apéndice 


\section{Bibliografía}

GÓMEZ S. Mario

1991 Didáctica de la Filosofía, Bogotá, Ed. USTA.

SANTIUSTE, Victor

1984 Didáctica de la Filosofía, Madrid, Ed. Narcea.

BONDY, Augusto S.

1967 Didáctica de la Filosofía, Lima, Ed. Arica.

PERELLÓ, Julio

1980 Apuntes de Didáctica General, Quito, I.S.S.

\section{APÉNDICE: Objetivos en la enseñanza de la filosofía}

\section{Lógica}

- Determinar la importancia de la Lógica como ciencia e instrumento básico para el razonamiento, a través de la dinámica del pensamiento, y su aplicación en la investigación científica.

- Emplear la sintaxis, la semántica y la práctica, en razonamiento lógico y matemático, para alcanzar no sólo la verdad, sino también la exactitud.

\section{2. Ética}

- Comprender que el hombre es un ente social que está íntimamente ligado a una vivencia de índole moral, sujeto a relaciones en las que debe tomar sus propias decisiones.

- Buscar mejores normas que le permitan lograr su bienestar social, mediante la reflexión crítica de los 
principios y doctrinas morales, en relación con el medio y los cambios de la sociedad.

- Discriminar actitudes que le permitan desenvolverse bien en las circunstancias de la vida humana, en la individual, familiar, socio-política y económica, mediante una valoración moral.

- Orientar a los alumnos en el análisis de los principios básicos que rigen la conducta humana en sus diversas actividades, a base del respeto de los valores éticos fundamentales.

- Propender a la consecución de la amistad, fraternidad y solidaridad con todos los pueblos, mediante la realización de los valores morales.

\section{Problemas filosóficos}

- Reconocer el valor y la influencia del pensamiento filosófico, en la resolución de todos los problemas que inquietan a la humanidad.

- Conocer las diversas teorías filosóficas que interpretan el origen de la materia, de la vida, del hombre, del universo, de la ciencia y otras inquietudes presentes.

- Desarrollar la capacidad de ordenamiento, sistematización y análisis de los conocimientos en cualquier campo de la actividad humana.

- Comprender el valor del pensamiento, fundamento básico en el proceso de las explicaciones y estudio de los fenómenos.

- Encontrar respuestas más justas en la problemática de la sociedad contemporánea.

De la "Programación curricular", Nivel medio. Lic. S. MARCILLO - Dra. R. TORRES R. 